\title{
The Prevalence and Genotype of Human Papillomavirus from Patients with Genital Warts in Eastern Guangdong Province
}

\author{
Zhao-Yun Luo ${ }^{1}$, Qiang Chen ${ }^{1}$, Hui Yang ${ }^{1}$, Min Lin ${ }^{1}$, Chan-Yu Chen ${ }^{2}$, Chun Yang ${ }^{2}$, \\ Li-Ye Yang ${ }^{1 *}$
}

\begin{abstract}
Background: Low-risk human papillomavirus (LR-HPV) infection is the main cause of genital warts. LRHPV genotypes 6 and 11 are associated with genital warts, but there have only been a few published studies about the genotype-specific prevalence of HPV in genital warts in China. The objective of our study was to assess the prevalence of HPV genotypes for clinical cases involving both men and women and to evaluate the potential benefit of a quadrivalent (genotypes 6, 11, 16, and 18) HPV vaccine in eastern Guangdong province of China. Materials and Methods: A total of 696 eligible patients with genital warts were enrolled during the period Aug 2009 through Oct 2014. Specimens were collected from genital warts, the HPV GenoArray test was used for HPV detection and genotyping, which could detect 21 HPV genotypes, including genotypes 6, 11, 16, and 18. Results: Among the 696 cases, 675 samples were successfully genotyped. The median age of patients was 32.1 years (range, 16-67 years). The most prevalent genotypes were HPV-6 (285/675, 42.2\%), HPV-11 (265/675, $39.3 \%)$, HPV-52 (52/675, 7.7\%), HPV-16(51/675, 7.56\%), HPV-81 (50/675, 7.40\%) and HPV-58 (37/675,5.48\%). Low-risk genotypes predominated, with a prevalence of $96.59 \%$. The cumulative prevalence of genotypes 6 and 11 was $78.7 \%(531 / 675)$, the cumulative prevalence of genotypes 16 and 18 was $11.6 \%(78 / 675)$, and the cumulative prevalence of genotypes $6,11,16$, and 18 was $82.5 \%$ (557/675). Conclusions: Our results provide strong evidence that, in eastern Guangdong, different from Western countries, the most prevalent low risk HPV genotypes in patients with genital warts are 6,11 and 81 . The quadrivalent HPV vaccine could prevent $82.5 \%$ of genital warts in eastern Guangdong.
\end{abstract}

Keywords: Human papillomavirus - genital warts - prevalence - China

Asian Pac J Cancer Prev, 16 (14), 5675-5679

\section{Introduction}

Cervical cancer is the second most common cancer in females worldwide, including China (Chen et al., 2012). Molecular epidemiological studies clearly indicate that certain types of human papillomavirus (HPV) are the principal cause of cervical cancer (Walboomers et al., 1999; de Villiers et al., 2004; Chen et al., 2012). Over 120 different types of HPVs have been identified, and approximately one-third of these types specifically infect the anogenital tract (de Villiers et al., 2004). Genital HPV types have been subdivided into low risk (LR) types which are the cause of external genital warts, and high risk (HR) types which have high oncogenic potential for progression to cervical cancer (de Villiers et al., 2004). Prevalence of HPV infection has been estimated to be about $17.7 \%$, varying between $6.52 \%$ and $20 \%$ depending on geography of China (Chen et al., 2012; Zhao et al., 2012). It is estimated that most females will be infected with HPV in their lifetime. Fortunately, HR-HPV infections typically last for 12-18 months and are eventually cleared completely by the immune system (Richardson et al., 2003). Approximately $10 \%$ of women fail to clear HPV infections, resulting in long-term persistent infections (Bodily et al., 2001). Persistence of HR-HPV infection is the single most important risk factor in the development of cervical intraepithelial neoplasia (CIN) (Walboomers et al., 1999; Bodily et al., 2001).

Specifically, high risk types 16 and 18 are the most common HPV types found in cervical cancers and account for about $70 \%$ of cervical cancers (Chen et al., 2012; Zhao et al., 2012). HPV-6 and HPV-11 are the most commonly detected LR-HPV genotypes in genital warts (Cremin et al., 2012; Aubin et al.,2008; Johnson et al., 2012). Up to date, several vaccines have been developed to prevent HPV infection. Gardisil is a quadrivalent vaccine containing four genotypes, namely 16,18, 6 and 11; and Cervarix (GSK) is a bivalent vaccine containing 16 and 18. In addition to providing excellent protection against the HPV types contained in the vaccine, Gardisil has 
also demonstrated significant cross-protection to other oncogenic types in both naïve and previously infected women (Mc Cormack eta 1., 2011).

At present in eastern Guangdong province of China, cervical lesions are screened by DNA-based testing for HR-HPV, and cervical exfoliated cells smear examinations is adjunct for the screening program, as this substantially increases the sensitivity of detecting neoplasia (Lin et al., 2008; Chen et al., 2012; Chen et al., 2012). In this study, we would like to present the distribution of HPV types in genital warts of a cohort of Chinese in Guangdong to contribute essential data for assessing the potential benefit of HPV vaccines containing HPV 6 and 11 .

\section{Materials and Methods}

\section{Patient recruitment and sample collection}

Chaozhou City located in easternmost Guangdong province of the People's Republic of China. From August 2009 to October 2014, the study was carried out in Chaozhou Central Hospital. The initial diagnosis of genital warts was made by gynaecologist, dermatologist, or urologist. A standardized sampling protocol was provided to each physician. One representative sample was collected from each patient. Biological samples were obtained from the most typical genital lesions (from coronal sulcus, glans penis, shaft, scrotum, vulva, vagina, perineum, and perianal skin) using a cotton swab (Hybribio Biotechnology Limited Corp.). The cotton swab was then placed in phosphate-buffered saline (PBS), and kept at $4{ }^{\circ} \mathrm{C}$ until further processing in laboratory. For females with cervical warts, specimens were collected from cervical exfoliated cells based on the method described previously (Chen et al., 2012; Lin et al., 2008; Li et al., 2011), samples of exfoliated cervical cells were collected using plastic cervical swabs. The sampler was inserted $1-1.5 \mathrm{~cm}$ into the endocervical canal and rotated 4-5 full turns in counter-clockwise direction. The tip containing cellular material was then placed into transport medium tube and stored at $4{ }^{\circ} \mathrm{C}$ immediately. All swabs and store bottle with specimen transport medium were from Hybribio Biotechnology Limited Corp., Chaozhou, China. The study was carried out with the approval of the ethical committee of Chaozhou Central Hospital, and patients consent was obtained for the collection of samples.

\section{HPV DNA extraction and HPV GenoArray test}

The swabs and supernatant were removed after the cells were centrifugated for 5 mins with relative centrifugal force of $960 \mathrm{~g}$. DNA was extracted from the sediments with alkaline lysis method Kits (Hybribio Biotechnology Limited Corp.) (Lin et al., 2008; Chen et al., 2012b). HPV GenoArray test was used for HPV detection and genotyping. Genotyping was done by DNA amplification, flow-through hybridization and gene chip by HybriMax (Hybribio Biotechnology Limited Corp., Chaozhou, China). Test was performed according to the manufacture's instructions. Detailed protocols for this assay had been described previously (Lin et al., 2008; Chen et al., 2012a; Chen et al., 2012b). The gene chip contained type-specific oligonucleotides immobilised on a nylon membrane and could identify $15 \mathrm{HR}-\mathrm{HPV}$ types $(16,18,31,33,35,39,45,51,52,53,56,58,59$, 66 and 68) and 6 LR-HPV types $(6,11,42,43,44$, and 81) (Lin et al., 2008). The final results were detected by colorimetric change on the chip under direct visualization (Lin et al., 2008; Li et al., 2011; Chen et al., 2012a; Chen et al., 2012b).

\section{Statistical analysis}

For age-specific HPV prevalence assessment, the actual age of each study subject was counted with the formula: (interview date - birthday) / 365.25 by Microsoft Excel software. Chi-squared tests were used to assess the statistical significance of any differences in prevalence. All data were analyzed using SPSS software version 16. $P$ values were two-sided, and statistical significance was accepted if the $P$ value was 0.05 or less. Quantitative data are expressed as mean (SD) and range.

\section{Results}

\section{Patient characteristics.}

The subjects of our study was carried out in Chaozhou Central Hospital and most of them were natives. The initial diagnosis of genital warts was made by gynaecologist, dermatologist, or urologist. Male genital warts were from coronal sulcus, glans penis, shaft, scrotum, perineum, and perianal skin and female warts were mostly from vulva, vagina and cervix. Of the 696 samples tested by the GenoArray assays, 675 (96.98\%) samples were found to have definable genotypes. 21 cases $(3.02 \%, 13$ males and 8 females) which were negative may not have had virus present, or negative for $\beta$-globin, or, had subdetectable levels on the genital warts, or may not have had genotypes that matched the panel covered by the GenoArray assays, were excluded from the following type distribution analysis.

The remaining 675 samples were successfully genotyped and were further analyzed, including 305 males, mean age $33.25 \pm 10.16$ (16-65 years), 370 females, mean age $31.06 \pm 10.06$, (17-67 years), the median age of patients was $32.05 \pm 10.16$ years (range, 16-67 years) for the entire group, the median age varied slightly between men and women (33.25 vs. 31.06 years;).

\section{HPV prevalence}

Presence of $1 \mathrm{HPV}$ genotype was only observed in $453(67.11 \%)$ of 675 cases, whereas $222(32.89 \%)$ cases involved infection with $\geq 2$ genotypes (Table 1). Multiple infections were seen in $29.19 \%$ of women and $37.38 \%$ of men, and single infection was more common in women than men $(p<0.01)$ (Table 1). On the basis of the lowrisk/high-risk genotype classification of previous studies (de Villiers et al., 2004; Chen et al., 2012a; Chen et al., $2012 b$ ), presence of $\geq 1$ low-risk HPV genotype was found in $652(96.59 \%)$ of 675 cases, and presence of $\geq 1$ high-risk genotype was found in 243 cases $(243 / 675,36 \%$ ) (Table $1)$. These proportions varied significantly with sex; the presence of $\geq 1$ low-risk genotype was similar between men and women (282/305, 92.46\% vs. 327/370, 88.38\%; 
Prevalence and Genotypes of Human Papillomaviruses from Patients with Genital Warts in Eastern Guangdong Province

$\mathrm{P}>0.05$ ) (Table 1). In contrast, presence of $\geq 1$ high-risk genotype was also similar between men and women $(120 / 305,39.34 \%$ vs. 124/370, 33.51\%; $p>0.05)$ (Table 1). $432(64 \%)$ of 675 genital wart samples demonstrated infection with $\geq$ low-risk genotype (without high-risk

Table 1. Distribution of HPV Types in 675 Genital Wart Specimens, According to the Low-risk And High-risk Classification

\begin{tabular}{lrrr}
\hline HPV types & $\begin{array}{c}\text { Male } \\
(\mathrm{n}=305)\end{array}$ & $\begin{array}{r}\text { Female } \\
(\mathrm{n}=370)\end{array}$ & $\begin{array}{c}\text { Total } \\
(\mathrm{n}=675)\end{array}$ \\
\hline Single-type infection & 191 & 262 & 453 \\
6 & 95 & 86 & 181 \\
11 & 70 & 90 & 160 \\
81 & 4 & 46 & 50 \\
16 & 1 & 2 & 3 \\
18 & 4 & 3 & 7 \\
$31 / 33 / 39 / 45 / 51 / 52 /$ & 11 & 22 & 33 \\
$53 / 58 / 59 / 66 / 42 / 43 / 44$ & 5 & 13 & 18 \\
Multiple-type infection & 114 & 108 & 222 \\
Two HPV types & 67 & 69 & 136 \\
6,11 & 3 & 2 & 5 \\
6,16 & 4 & 2 & 6 \\
6,18 & 2 & 2 & 4 \\
11,16 & 3 & 5 & 8 \\
11,18 & 4 & 2 & 6 \\
16, 18 & 0 & 1 & 1 \\
Other combination & 51 & 55 & 106 \\
Three HPV types & 26 & 25 & 51 \\
Four or more HPV types & 16 & 14 & 30 \\
\hline
\end{tabular}

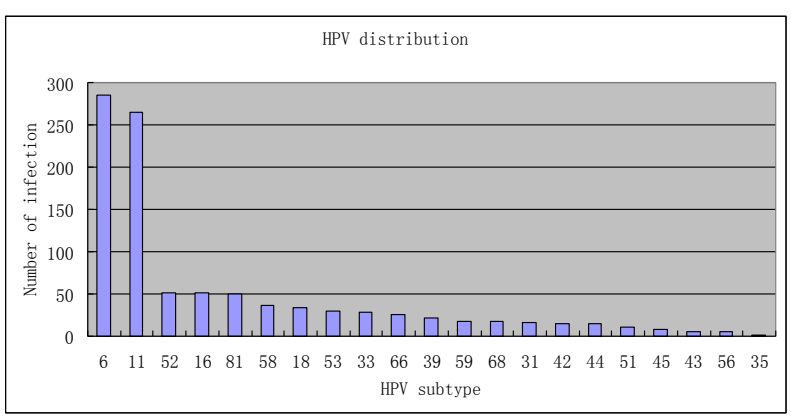

Figure 1. Distribution of HPV types among 675 successfully genotyped genital warts. HPV types were counted regardless of the status of single- or multipletype infections. 222 specimens with more than one HPV type were counted more than once

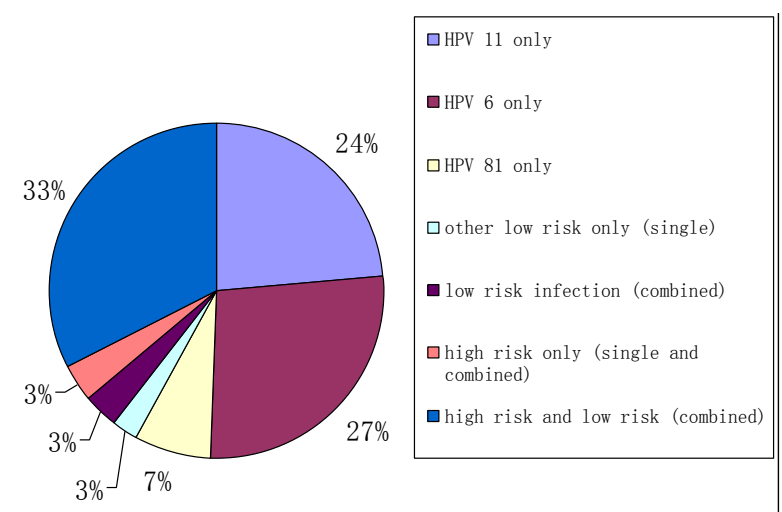

Figure 2. Distribution of HPV types in 675 successfully genotyped genital warts according to HPV6/11/81 status genotypes); 23 (3.4\%) demonstrated infection $\geq 1$ highrisk genotype (without low-risk genotypes), whereas 220 (32.6\%) demonstrated co-infection with low-risk and high-risk genotypes (Table 1).

\section{Prevalence of specific HPV genotypes}

The most frequently encountered HPV genotypes of 675 samples were as follows, by decreasing order of frequency: HPV-6 (285/675, 42.22\%), HPV-11 (265/675, 39.26\%), HPV-52 (52/675, 7.7\%), HPV-16 (51/675, 7.56\%), HPV-81 (50/675, 7.4\%) and HPV-58 (37/675, 5.48\%) (Table 1). The distribution of HPV types, regardless of single- or multiple-type infection, among the $675 \mathrm{HPV}$-positive samples was shown in Figure 1. In women, the 6 commonest types were HPV-11, HPV-6, HPV-81, HPV-52, HPV-16 and HPV-58, whereas in men, the 6 commonest types were HPV-6, HPV-11, HPV-16, HPV-52, HPV-18, and HPV-58 (Table 1).

Low-risk HPV genotypes 6 and/or 11 were found more frequently in men than in women $(267 / 305,87.54 \%$ vs.263/370, 71.08\%; $P<0.01)$.

Conversely, high-risk HPV genotypes 16 and/or 18 distributed equally in men and women (42/305, 13.77\%\% vs. 39/370, 10.54; $p>0.05)$.

Interestingly, low-risk HPV genotypes 81 was more common among women than among men $(65 / 370,17.57 \%$ vs.17/305, 5.57\%; $p<0.001)$.

Furthermore, single-type 81 infection was more common among women than among men (46/370, $12.43 \%$ vs. 4/305, 1.31\%; $p<0.01)$.

The distribution of HPV types was analyzed with respect to HPV6 and 11, to assess the potential coverage for genital warts with vaccines containing these types (Figure 2). HPV-6 and/or HPV-11 (alone or in association with each other) were found in $51.26 \%$ (346/675) of cases. These 2 genotypes was detected in combination with other HPV types in $27.26 \%(184 / 675)$ of genital wart cases, yielding an overall prevalence of HPV-6 and/or HPV-11 infection of 78.52\% (530/675) (Figure2).

Similarly, HPV-16 and/or HPV-18 (alone or in association with each other) were observed in $1.63 \%$ (11/675) of specimens. HPV-16 and/or HPV-18 were further detected in combination with other HPV genotypes in $9.93 \%$ (67/675) of genital wart cases, yielding an overall prevalence of infection with HPV-16 and/or HPV-18 of $11.55 \%$ (78/675). HPV-6, HPV-11, HPV-16, and/or HPV18 (alone or in association with each other) were observed in $56.6 \%(382 / 675)$ of all cases. Finally, at least 1 of these 4 genotypes (alone or in combination with other genotypes) was found in $82.52 \%(557 / 675)$ of cases.

\section{Discussion}

Human papillomavirus (HPV) infection is a common sexually transmitted infection worldwide. HPV may cause several reproductive tract diseases, including genital warts and cervical cancer, the later is the most serious health problem due to persistent HR-HPV infection. Although cervical cancer showed a declining trend over the past 3 decades in China, it remains a major health burden in Chinese females especially women living in rural area. The 
disease burden is believed to be underestimated given the relatively high HPV prevalence shown in recent studies (Chan et al., 2009).

Up to date, about 120 kinds of HPV subtypes have been characterized, above 40 kinds of subtypes was associated with genital tract infection, they were classified into high risk and low risk based on their carcinogenic capability. The pathogen of genital warts was low risk HPV (LR-HPV). HPV6, 11, 42, 43, 44 and 81 was generally considered as low-risk, the main subtypes for genital warts, whereas HPV16, 18, 31, 33, 35, 39, 45, $51,52,56,58,59,68,53$ and 66 were classified as highrisk (de Villiers et al., 2004). Generally, HR-HPV could incorporated into genome of host, persistent infection or recurrent infection of HR-HPV was the main factor of cervical cancer, the pathogenic ability of HPV was closely associated with HPV subtypes(Walboomers et al., 1999), therefore, HPV subtyping and evaluation were very important for clinical practice.

To obtain the first data concerning HPV and genital warts in eastern Guangdong, 696 genital wart tissue specimens were tested for the presence of HPV DNA using GenoArray test, the samples from 675 cases of genital warts were successfully genotyped for HPV. Single infection ratio was $67.11 \%(453 / 675)$, mainly HPV11 and 6 , multiple infection was $32.89 \%$, mainly was mixed infection of HR and LR, double and triple infection was the main multiple infections. HPV-6 or HPV-11 was detected in $78.67 \%$ of genital warts studied, which was in agreement with the results of similar recent studies (Aubin et al., 2008; Johnson et al., 2012; Chen et al., 2012b). This study showed that HPV subtypes infection of genital warts of our area were mainly low risk HPV11 and HPV6, consistent to previous study (Chan et al., 2009; Cremin et al., 2012), possibly due to common warts sample of genitals.

According to the recent phylogenetic classification (de Villiers et al., 2004), HPV-81 belongs to the alpha-HPV species 3 together with other HPV genotypes: HPV-72, HPV-83, HPV-84 and HPV-86. Single infection of HPV 81 was more frequently found in the females than in the males in our study. This demonstrated that HPV 81 was more prone to induce epithelial lesion (genital warts) in women other than in males, another explanation was that HPV81 could be eliminated by males quickly, the mechanism underlining it was not clear yet. According to the published data, our study is the first to report the presence of 81 as a single HPV genotype in genital warts tissue specimens, giving this particular HPV genotype a more prominent place in the etiopathogenesis of genital warts than was supposed before.

In our study, which was performed exclusively in individuals without any apparent immunodeficiency, 222 out of 675 genital wart specimens contained more than one HPV genotype. In addition to HPV-6 or HPV-11, which were detected in $28.15 \%$ (190/675) samples with mixed HPV infection (Table 1, Figure 1). Our results agreed with the results of similar recent studies, the prevalence of simultaneous infection with different HPV genotypes (mixed HPV infection), ranged from $30 \%$ to $54 \%$, mainly depending on the immune status of the individuals included in the study and the selection of method used for HPV genotyping (Aubin et al., 2008; Chan et al., 2009; Cremin et al., 2012).

119 of 675 samples had one or more of the HR genotypes 31 , 33, 45, 52, 58 with or without co-infection with 16 and 18. As indicated by Brown et al (Brown et al., 2009), these HR types have $80 \%$ homology with genotypes 16 and 18 and as a clade, the additive efficacy of Gardisil is $25 \%$ on a population level. Our study population infected with these additional genotypes should therefore benefit from cross-protection with Gardisil.

The cumulative prevalence of genotypes $6,11,16$, and 18 was $82.52 \%(557 / 675)$ in our study. Therefore, Gardisil, the quadrivalent vaccine, would have prevented infections in $82.52 \%$ of our patient population. HPV16 and/or 18, high-risk types being included in currently available vaccine Cervarix (GSK), were found in genital warts specimens, with a majority of which existed as coinfections with HPV6 and/or 11, would have prevented infections in $11.55 \%$ of our patient population.

In conclusion, our study showed that HPV can be found in virtually all tissue specimens of genital warts obtained from patients in eastern Guangdong. This finding suggests that, if the recently approved quadrivalent HPV vaccine proves to be as effective in preventing HPV infections in males as it has proved in females (Brown et al., 2009), this vaccine could prevent the great majority of incidental GWs in males in eastern Guangdong.

\section{References}

Aubin F, Pretet JL, Jacquard AC, et al (2008). Human papillomavirus genotype distribution in external acuminata condylomata: a Large French National Study (EDiTH IV). Clin Infect Dis, 47, 610-5.

Bodily J,Laimins LA(2011). Persistence of human papillomavirus infection: keys to malignant progression. Trends Microbiol, 19, 33-9.

Brown DR, Kjaer SK, Sigurdsson K, et al (2009).The impact of quadrivalent human papillomavirus (HPV; types 6,11 , 16, and 18) L1 virus-like particle vaccine on infection and disease due to oncogenic nonvaccine HPV types in generally HPV-naive women aged 16-26 years. J Infect Dis, 199, 926-35.

Chan PK, Luk AC, Luk TN, et al (2009). Distribution of human papillomavirus types in anogenital warts of men. J Clin Virol, 44, 111-4.

Chen Q, Xie LX, Qing ZR, et al (2012). Epidemiologic characterization of human papillomavirus infection in rural chaozhou, eastern guangdong province of China. PLoS One, 7, 32149 .

Chen Q, Luo ZY, Lin M, et al (2012). Prevalence and genotype distribution of human papillomavirus infections in women attending hospitals in Chaozhou of Guangdong province. Asian Pac J Cancer Prev, 13, 1519-24.

Cremin S, Menton JF, Canier L, Horgan M, Fanning LJ (2012). The prevalence and genotype of human papillomavirus on cervical samples from an Irish female population with external genital warts. Hum Vaccin Immunother, 8, 916-20.

de Villiers EM, Fauquet C, Broker TR, Bernard HU, zur Hausen $\mathrm{H}$ (2004). Classification of papillomaviruses. Virol, 324, $17-27$.

Huang Y, Lin M, Luo ZY, et al (2013). Low prevalence of HPV in male sexual partners of HR-HPV infected females and low 

concordance of viral types in couples in eastern guangdong. Asian Pac J Cancer Prev, 14, 1755-60.

Johnson AM, Mercer CH, Beddows S, et al (2012). Epidemiology of, and behavioural risk factors for, sexually transmitted human papillomavirus infection in men and women in Britain. Sex Transm Infect, 88, 212-7.

Li J, Kang LN, Qiao YL (2011). Review of the cervical cancer disease burden in mainland China. Asian Pac J Cancer Prev, 12, 1149-53.

Lin M, Yang LY, Li LJ, et al (2008). Genital human papillomavirus screening by gene chip in Chinese women of Guangdong province. Aust N Z J Obstet Gynaecol, 48, 189-94.

McCormack PL, Joura EA (2011). Spotlight on quadrivalent human papillomavirus (types $6,11,16,18$ ) recombinant vaccine $\left(\right.$ Gardasil $\left.^{\circledR}\right)$ in the prevention of premalignant genital lesions, genital cancer, and genital warts in women. Bio Drugs, 25, 339-43.

Richardson H, Kelsall G, Tellier $P$, et al (2003). The natural history of type-specific human papillomavirus infections in female university students. Cancer Epidemiol Biomarkers Prev, 12, 485-90.

Walboomers JM, Jacobs MV, Manos MM, et al (1999). Human papillomavirus is a necessary cause of invasive cervical cancer worldwide. J Pathol, 189, 12-9.

Zhao FH, Lewkowitz AK, Hu SY, et al (2012). Prevalence of human papillomavirus and cervical intraepithelial neoplasia in China: a pooled analysis of 17 population-based studies. Int J Cancer, 131, 2929-38. 